\title{
Blind Chip-Rate Multiuser Equalisation
}

\author{
Stephan Weiss and Mahmoud Hadef \\ Communications Research Group \\ School of Electronics \& Computer Science \\ University of Southampton, UK \\ Email: $\{$ s.weiss,mh02r\}@ecs.soton.ac.uk
}

\begin{abstract}
This paper addresses blind multiuser detection in a DS-CDMA downlink channel. The synchronous users are separated by re-establishing orthogonality of their spreading sequences in a common chip-rate equaliser. The adaptation algorithm can be based either on a constant modulus (CMA) criterion of the various users, or on a decision directed (DD) scheme. In either case, a stochastic gradient descent algorithm will result, as derived, in a multiple error filtered-X LMS type approach, whereby the equaliser input to both the multichannel CMA or DD algorithm are replaced by spreading code filtered versions. Adaptation examples are given underlining some of the characteristics of the proposed algorithms.
\end{abstract}

\section{Introduction}

This contribution is aimed at exploiting the power of a programmable receiver such as fund in a software defined radio in order to design and implement signal processing techniques for advanced receiver functionality. Specifically, we consider a DS-CDMA downlink system in Fig. 1 with multiple synchronous users, which for simplicity are assumed to have the same data rate. Each user $u_{l}[n], l=0(1) N-1$, is code multiplexed using Walsh sequences of length $N$ extracted from a Hadamard matrix $\mathbf{H}$. The code multiplexed signal is further scrambled by multiplication with $c[m]$ prior to transmission over a dispersive channel $h[m]$ with additive noise $v[m]$.

The dispersive channel $h[m]$ destroys the orthogonality of the Walsh codes, such that direct decoding of the received signal $r[m]$ will lead to multiple access interference. Hence, much research concentrated on symbol-rate equalisers, such as in $[1,2,3]$. The ability to apply computationally complex signal processing tasks at higher sampling rates has recently lead to attractive solutions, whereby the equaliser is operated at the chip-rate [4]. In the later, the chip-rate equaliser $w[\mathrm{~m}]$ is updated by comparing its output to a spread and scrambled training

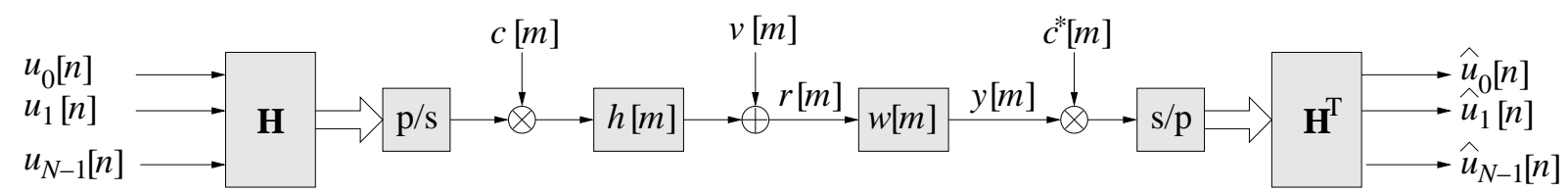

Fig. 1: CDMA modulation and demodulation of user signals $u_{l}[n], l=0(1) N-1$, incorporating a dispersive channel $h[m]$ and a chip-rate equaliser $w[m]$. 
sequence. Alternatively, receiver can operate in a decision directed mode, whereby the detected symbols are re-spread, scrambled, and utilised to form an error with which $w[\mathrm{~m}]$ is corrected.

The method in [4] requires substantial training, whereby the suggested structure can be switched into a decision directed stage. Decision errors however lead to error propagation with lethal consequences for the convergence of the chip-rate equaliser and subsequently the detected user sequences $\hat{u}_{l}[n], l=0(1) N-1$. Therefore, in this paper we address a way of blindly adapting the chip-rate equaliser $w[m]$.

\section{Proposed Structure}

We first derive the detected user signals $\hat{u}_{l}[m]$ as a function of the chip-rate equaliser $w[m]$. Based on this, we state suitable cost functions based on which the equaliser can be adapted.

\subsection{Received Signals}

The short codes for multiplexing the users are assumed to be Walsh sequences taken from a Hadamard matrix $\mathbf{H}^{\mathrm{T}}$. For the decoding, the Walsh sequences are time reversed,

$$
\mathbf{H}^{\mathrm{T}}=\left[\begin{array}{llll}
\mathbf{h}_{0} & \mathbf{h}_{1} & \cdots & \mathbf{h}_{N-1}
\end{array}\right]^{\mathrm{T}} .
$$

The $l$ th user is thus decoded as

$$
\begin{aligned}
& \hat{u}_{l}[n]=\mathbf{h}_{l}^{\mathrm{T}} \cdot\left[\begin{array}{cccc}
c^{*}[n N] & & & \mathbf{0} \\
& c^{*}[n N-1] & & \\
& & \ddots & \\
\mathbf{0} & & & c^{*}[n N-N+1]
\end{array}\right] \cdot\left[\begin{array}{c}
y[n N] \\
y[n N-1] \\
\vdots \\
y[n N-N+1]
\end{array}\right] \\
& =\tilde{\mathbf{h}}_{l}^{\mathrm{T}}[n N] \cdot\left[\begin{array}{cccc}
\mathbf{w}^{\mathrm{H}} & & & \\
& \mathbf{w}^{\mathrm{H}} & & \\
& & \ddots & \\
& & & \mathbf{w}^{\mathrm{H}}
\end{array}\right] \cdot\left[\begin{array}{c}
r[n N] \\
r[n N-1] \\
\vdots \\
r[n N-L-N+1]
\end{array}\right] \\
& =\mathbf{w}^{\mathrm{H}} \cdot\left[\begin{array}{cccc}
\tilde{\mathbf{h}}_{l}^{\mathrm{T}}[n N] & & & \\
& \tilde{\mathbf{h}}_{l}^{\mathrm{T}}[n N] & & \\
& & \ddots & \\
& & & \tilde{\mathbf{h}}_{l}^{\mathrm{T}}[n N]
\end{array}\right] \cdot\left[\begin{array}{c}
r[n N] \\
r[n N-1] \\
\vdots \\
r[n N-L-N+2]
\end{array}\right] \\
& =\mathbf{w}^{\mathrm{H}} \mathbf{H}_{l}[n N] \mathbf{r}_{n N}
\end{aligned}
$$

with $\mathbf{w} \in \mathbb{C}^{L}, \mathbf{H}_{l}[n N] \in \mathbb{Z}^{L \times(N+L-1)}$ and $\mathbf{r}_{n N} \in \mathbb{C}^{N+L-1}$. The long spreading code $c^{*}[m]$ has been absorbed into a time varying short code vector $\tilde{\mathbf{h}}^{\mathrm{T}}[n]$.

\subsection{CMA Cost Function}

Assuming that the user signals $u_{l}[m]$ are constant modulus signals, such as BPSK, QPSK, or 8-PAM, with a modulus $\gamma$, we can formulate a cost function comprising $N$ terms

$$
\xi_{\mathrm{CMA}}=\mathcal{E}\left\{\sum_{l=0}^{N-1}\left(\gamma^{2}-\left|\hat{u}_{l}[n]\right|^{2}\right)^{2}\right\},
$$




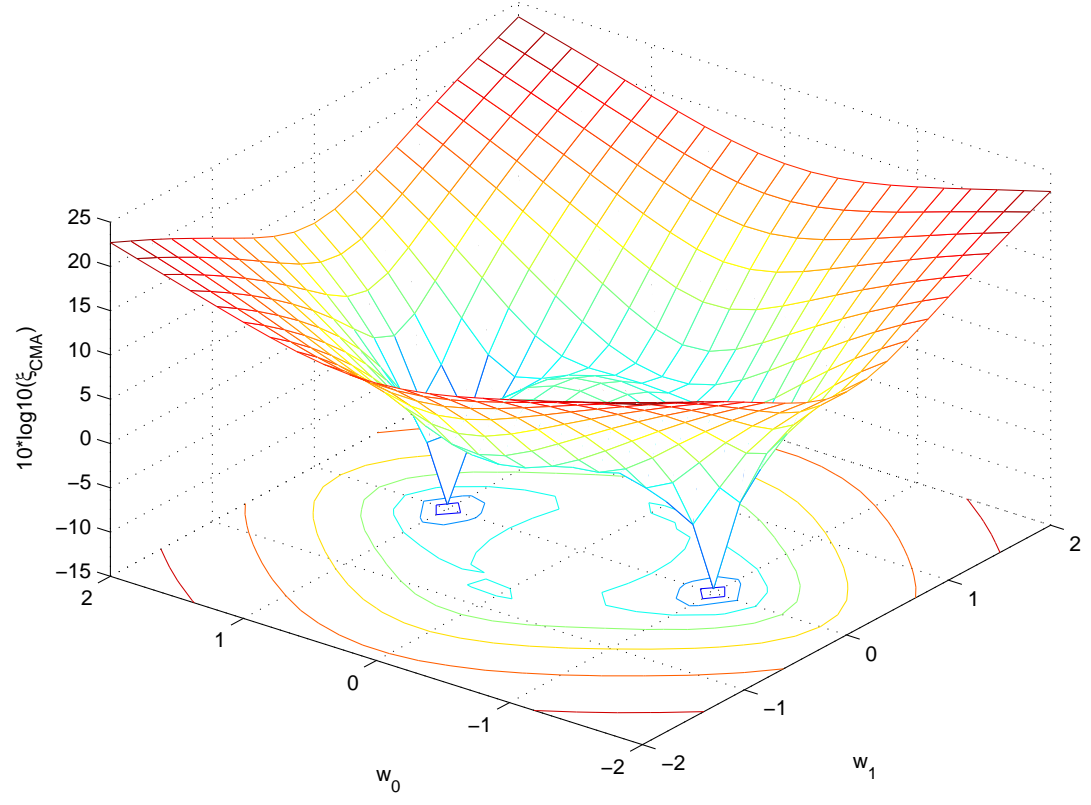

Fig. 2: CMA cost function in dependency of $\mathbf{w}=\left[\begin{array}{ll}w_{0} & w_{1}\end{array}\right]^{\mathrm{T}}$, which is constrained to be real.

whereby

$$
\mathbf{w}_{\mathrm{Opt}, \mathrm{CMA}}=\arg \min _{\mathbf{w}} \xi_{\mathrm{CMA}}
$$

which forces each received user symbol $\hat{u}_{l}[n]$ onto a constant modulus. Each term in the cost function accounts for the misadjustment of the $l$ th user in the system. The shape of this cost function is not concave, since the optimal solution in the sense of minimising (6) is ambiguous with a manifold of solutions due to an indeterminism in phase rotation.

An example for $\xi_{\mathrm{CMA}}$ with $N=4$ users employing QPSK over a distortionless noise-free channel in dependency of $\mathbf{w}$ with $L=2$ is given in Fig. 2. There are two symmetric solutions $\mathbf{w}_{\text {opt,CMA }}=\left[\begin{array}{ll} \pm 1 & 0\end{array}\right]^{\mathrm{T}}$ only, since $\mathbf{w}$ is restricted to be real. Note that a solution $\mathbf{w}=\left[\begin{array}{ll}0 & \pm 1\end{array}\right]^{\mathrm{T}}$ would not synchronise the codes correctly and therefore has a large cost function value. Also, the cost function can be seen to have flat points in between the minima.

\subsection{Decision Directed Cost Function}

By employing a non-linearity $q(\cdot)$ that maps its input onto the permitted constellation points by means of suitable decision thresholds, a decision directed cost function $\xi_{\mathrm{DD}}$ can be formulated,

$$
\xi_{\mathrm{DD}}=\mathcal{E}\left\{\sum_{l=0}^{N-1}\left(q\left(\hat{u}_{l}[n]\right)-\hat{u}_{l}[n]\right)^{2}\right\}
$$

whereby

$$
\mathbf{w}_{\mathrm{Opt}, \mathrm{DD}}=\arg \min _{\mathbf{w}} \xi_{\mathrm{DD}}
$$

An example for $\xi_{\mathrm{DD}}$ with $N=4$ users employing QPSK over a distortionless noise-free channel in dependency of $\mathbf{w}$ with $L=2$ is given in Fig. 3. There are two symmetric solutions $\mathbf{w}_{\text {opt,DD }}=\left[\begin{array}{ll} \pm 1 & 0\end{array}\right]^{\mathrm{T}}$; again note that $\mathbf{w}=\left[\begin{array}{ll}0 & \pm 1\end{array}\right]^{\mathrm{T}}$ would not synchronise the codes. 


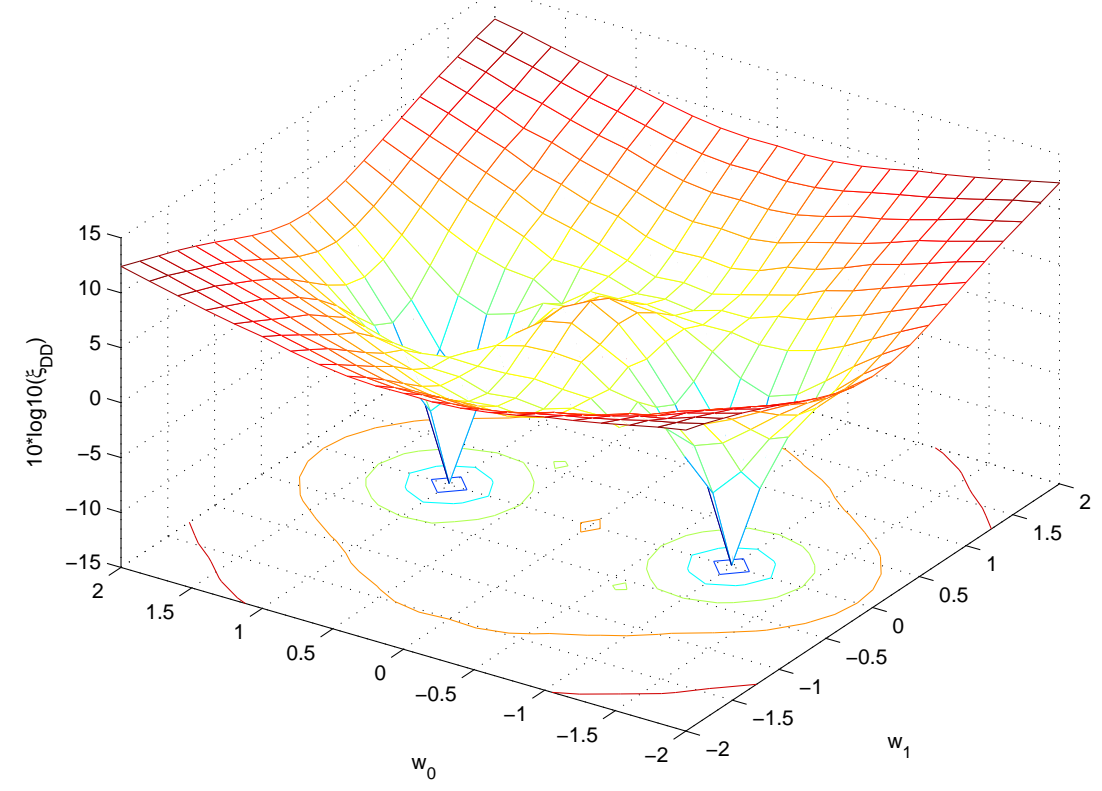

Fig. 3: DD cost function in dependency of $\mathbf{w}=\left[\begin{array}{ll}w_{0} & w_{1}\end{array}\right]^{\mathrm{T}}$.

\section{Stochastic Gradient Algorithms}

A simple stochastic gradient descent update rule for $w[\mathrm{~m}]$ can be found by calculating the gradient of an instantaneous cost function, i.e. omitting the expectation operator in (6) and (8). The resulting terms are then minimised w.r.t. $\mathbf{w}$ to obtain instantaneous estimates of the cost function gradient $\nabla \hat{\xi}\left(\mathbf{w}_{n}\right)$, leading to the stochastic gradient update

$$
\mathbf{w}_{n+1}=\mathbf{w}_{n}-\mu \nabla \hat{\xi}\left(\mathbf{w}_{n}\right)
$$

where $\mu$ is the step size. The introduction of gradient noise through inaccurate estimates of the true underlying statistics into the update routine can be expected to assist the system in recovering from flat points in the cost functions as seen in Figs. 2 and 3.

\subsection{Stochastic Gradient CMA}

Based on (6) with expectations dropped, we apply complex vector calculus [5]:

$$
\begin{aligned}
\frac{\partial}{\partial \mathbf{w}^{*}} \hat{\xi}_{\mathrm{CMA}} & =-2 \sum_{l=0}^{N-1}\left[\left(\gamma^{2}-\left|\hat{u}_{l}[n]\right|^{2}\right) \frac{\partial}{\partial \mathbf{w}^{*}} \hat{u}_{l}[n] \hat{u}_{l}^{\mathrm{H}}[n]\right] \\
& =-2 \sum_{l=0}^{N-1}\left[\left(\gamma^{2}-\left|\hat{u}_{l}[n]\right|^{2}\right) \mathbf{H}_{l}[n N] \mathbf{r}_{n N} \mathbf{r}_{n N}^{\mathrm{H}} \mathbf{H}_{l}^{\mathrm{H}}[n N] \mathbf{w}\right] \\
& =-2 \sum_{l=0}^{N-1}\left[\left(\gamma^{2}-\left|\hat{u}_{l}[n]\right|^{2}\right) \mathbf{H}_{l}[n N] \mathbf{r}_{n N} \hat{u}_{l}^{*}[n]\right]
\end{aligned}
$$

We refer to this algorithm as a multiple error filtered-X constant modulus algorithm (CMA) which structurally similar to a multiple-error filtered-X LMS algorithm [6], i.e. the equaliser input fed usually into the CMA algorithm is replaced by a version filtered with the short codes. 


\subsection{Stochastic Gradient with Decision Directed Updating}

The following derivation is based on the assumption that $q\left(\hat{u}_{l}[n]\right)$ is independent of $\mathbf{w}$ :

$$
\begin{aligned}
\frac{\partial}{\partial \mathbf{w}^{*}} \hat{\xi}_{\mathrm{DD}} & =-2 \sum_{l=0}^{N-1}\left[\left(q\left(\hat{u}_{l}[n]\right)-\hat{u}_{l}[n]\right) \frac{\partial}{\partial \mathbf{w}^{*}} \hat{u}_{l}[n]\right] \\
& =-2 \sum_{l=0}^{N-1}\left[\left(q\left(\hat{u}_{l}[n]\right)-\hat{u}_{l}[n]\right) \mathbf{H}_{l}[n N] \mathbf{r}_{n N}\right]
\end{aligned}
$$

Again, the term $\mathbf{H}_{l} \mathbf{r}_{n N}$ means that the input $r[m]$ to the chip-rate equaliser needs to be filtered by the Walsh codes $\mathbf{H}$ before being fed into a standard DD multichannel stochastic gradient equaliser update.

\section{Results}

We assume a channel impulse response at chip rate with coefficients $\mathbf{c}=\left[\begin{array}{lll}1 & .4-.3 j & .1\end{array}\right], N=4$ QPSK modulated users and an equaliser length of $L=10$ coefficients. The dispersive channel is assumed noiseless. Below we present and discuss the results using the algorithms derived in Sec. 3.

\subsection{CMA Experiment}

Using the multiple error filtered-X constant modulus algorithm, an example for a learning curve with a suitable step size $\mu$ is shown in Fig. 4(bottom), with the weight trajectories given in Fig. 4(top). The adaptation is initialised with the second coefficient in the weight vector set to unity, while the optimal response will need to place the maximum coefficient in the first vector element. The adapted response is a complex rotation of the ideal response, such that the received constellation pattern will be rotated as well, as can be seen in Fig. 5. Adaption is switched on at $n=0$, but the adaptation curve shows a slow initial convergence typical of CMA algorithms [7].

\subsection{DD Experiment}

The results for the decision directed stochastic gradient algorithm version are shown in Fig. 6 . The finite steady-state MSE is due to truncation of the ideal equaliser response after $L=10$ coefficients.

\section{Discussion and Conclusion}

We have presented a novel blind and fast converging multiple-error CMA algorithm updating a chip-rate equaliser which can be used in downlink CDMA system. Although not shown here, the introduction of channel noise will affect the final MSE, but both DD and CMA algorithm versions have demonstrated robustness towards noise in extensive simulations down to a channel SNR of about $5 \mathrm{~dB}$. The benefit of the presented method is a fairly low-cost algorithm 
and quick convergence in a DS-CDMA downlink scenario, and is an attractive alternative to constrained interference canncellation schemes [8] or multiuser CMA algorithm either without dispersion $[9,10]$ or without spreading [11].

\section{References}

[1] M. Honig, U. Madhow, and S. Verdu, "Blind Adaptive Multiuser Detection," IEEE Transactions on Information Theory, vol. 41, no. 4, pp. 944-960, July 1995.

[2] F. Petre, M. Moonen, M. Engels, B. Gyselinckx, and H.D. Man, "Pilot-Aided Adaptive CHip Rate Equalizer Receiver for Interference Suppression in DS-CDMA Forward Link," in IEEE Vehicular Technology Conference, Sept. 2000, vol. 1, pp. 303-308.

[3] M. D. Krauss, T. P. amd Zoltowski and G. Leus, "Simple MMSE Equalizers for CDMA Downlink to Restore Chip Sequence: Comparison to Zero-Forcing and Rake," in Proc. IEEE International Conference on Acoustics, Speech, and Signal Processing, June 2000, vol. 5, pp. 2865-2868.

[4] P. Schniter and A. R. Margetts, "Adaptive Chip-Rate Equalization of Downlink Multirate Wideband CDMA," in Asilomar Conference on Signals, Systems, and Computers, Monterey, CA, November 2002, pp. 1228-1232.

[5] T. K. Moon and W. C. Stirling, Mathematical Methods and Algorithms, Prentice Hall, Upper Saddle River, NJ, 1999.

[6] S.J. Elliott, I.M. Stothers, and P.A. Nelson, "A Multiple Error LMS Algorithm and its Application to the Active Control of Sound and Vibration," IEEE Transactions on Acoustics, Speech and Signal Processing, vol. Vol.35, no. No.10, pp. pp.1423-1434, 1987.

[7] C. R. Johnson, P. Schniter, T. J. Endres, J. D. Behm, D. R. Brown, and R. A. Casas, "Blind Equalization Using the Constant Modulus Criterion: A Review," Proceedings of the IEEE, vol. 86, no. 10, pp. 1927-1950, October 1998.

[8] R. Schober, W. H. Gerstacker, and L. H. J. Lampe, "Comparison of MOE and blind LMS," IEEE Communications Letters, vol. 7, no. 5, pp. 204-206, May 2003.

[9] K. Fukawa and H. Suzuki, "Orthogonalizing Matched Filter (OMF) Detection for DSCDMA Mobile Radio Systems," in IEEE Global Telecommunications Conference, 1994, vol. 1, pp. 385-389.

[10] Y. Huang and P. M. Djurić, "Multiuser Detection of Synchronous Code-Division MultipleAccess Signals by Perfect Sampling," IEEE Transactions on Signal Processing, vol. 7, no. 50, pp. 1724-1734, July 2002.

[11] C. Papadias and A. Paulraj, "A Constant Modulus Algorithm for Multiuser Signal Separation in Presence of Delay Spread Using Antenna Arrays," IEEE Signal Processing Letters, vol. 4, no. 6, pp. 178-181, June 1997. 

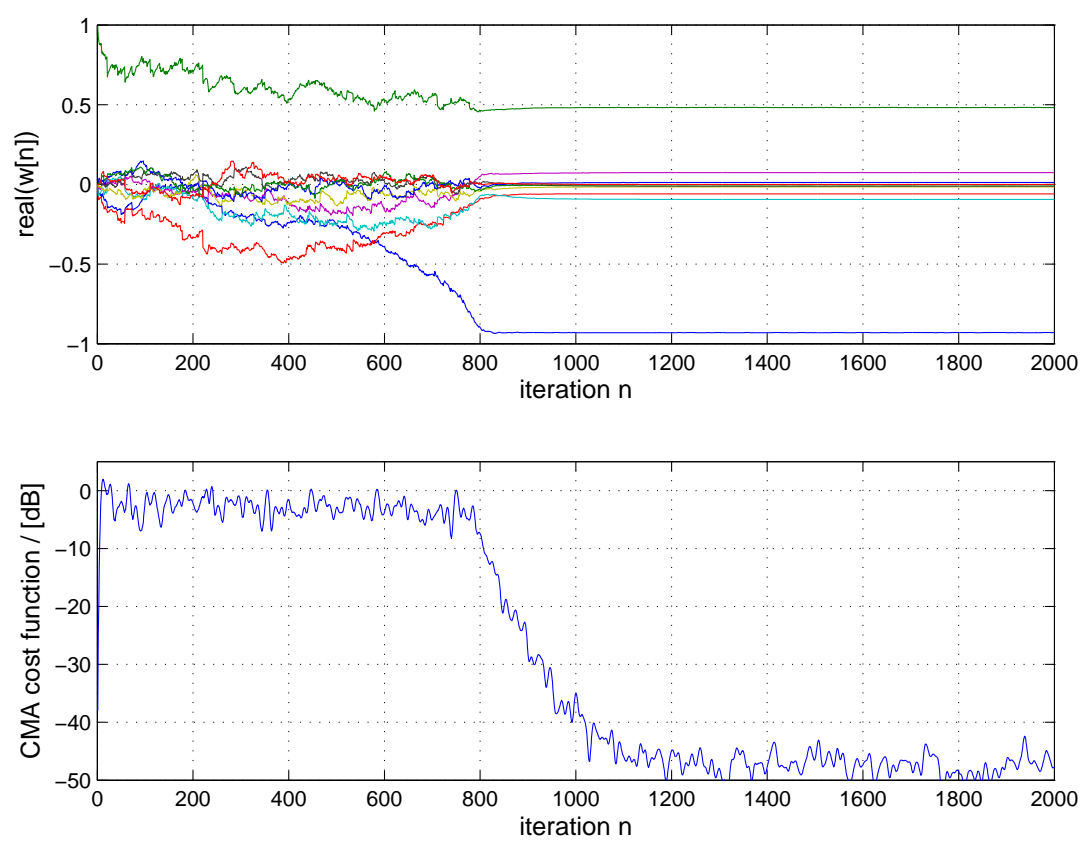

Fig. 4: (top) weight trajectories and (bottom) learning curve of the CMA experiment.

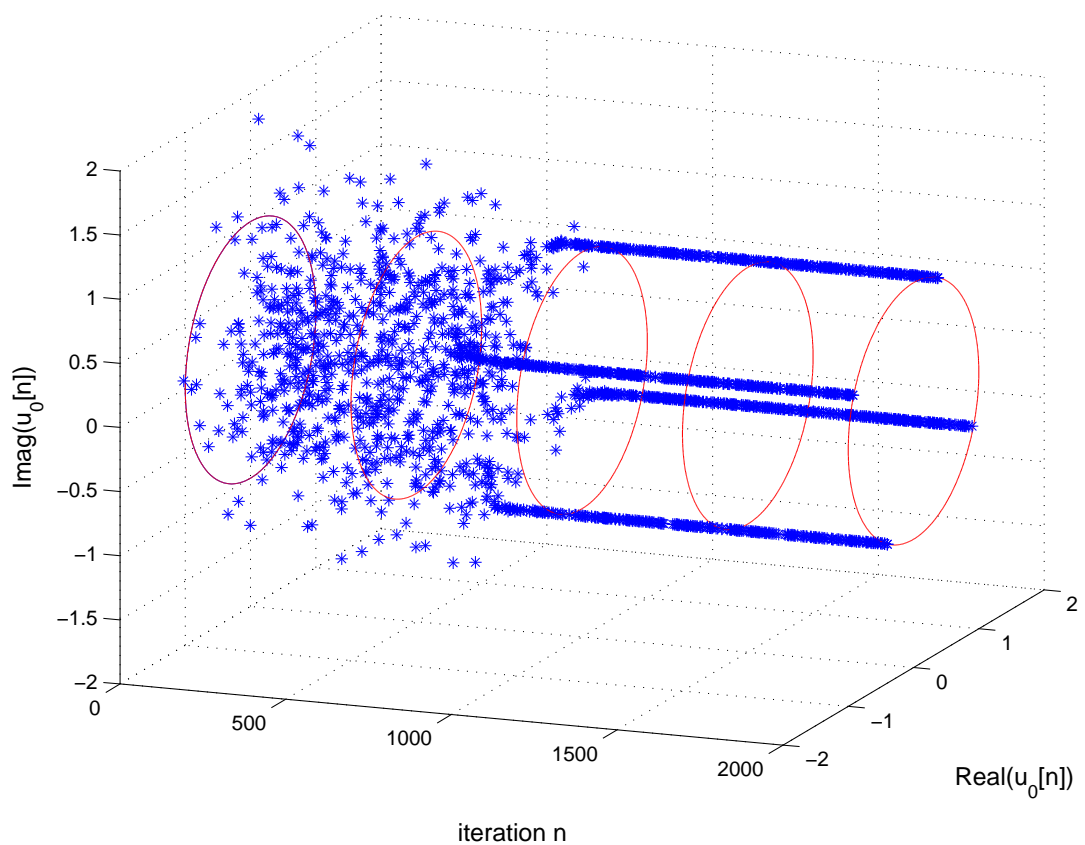

Fig. 5: Evolution of the received constellation pattern of user $l=0$ with the CMA algorithm. 

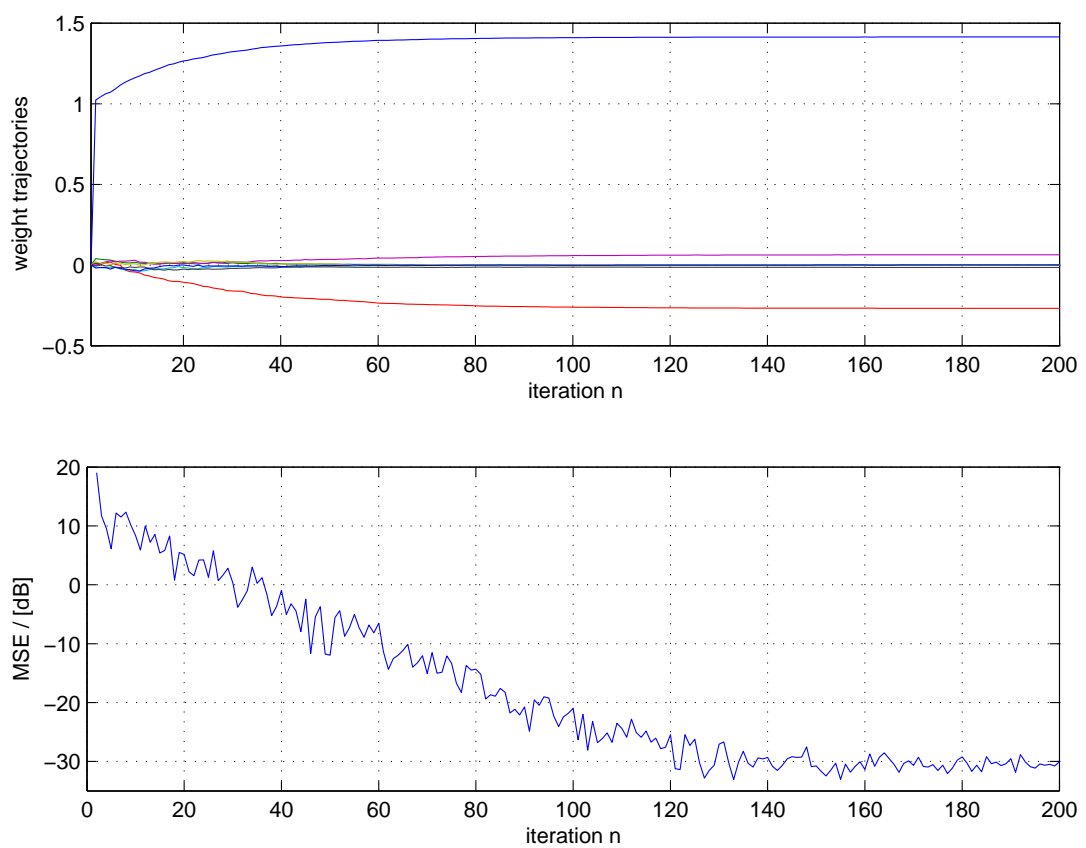

Fig. 6: (top) weight trajectories and (bottom) learning curve of the DD experiment. 